\title{
Result Assessment to Intrusion Detection System using Factors Analysis in MANET
}

\author{
Poonam Choubey \\ Shri Vaishnav Institute of \\ Science Indore (M.P.)
}

\author{
Rupali Bhartiya \\ Shri Vaishnav Institute of \\ Science Indore (M.P.)
}

\begin{abstract}
The idea of MANET is basically definite quality because of its unique courses of action and gets the chance to take part. Among different structures which are used in remote methods, flexible ad hoc system is seen as a potential region of work. This system is managed by own resources itself, along these nodes the behavior made for supporting this environment is besides light weighted. When this is a basic functionality has been arrangements which give a basic zone for finding attacker to control the working of the structure and shows effective conduct to avoid interruptions. Over the period of time, particular techniques had been proposed to update the energy issues of recognizing use in MANET. The main idea is to assess effective transmission and each one of the objectives is to make the system full proof which controls the conditions now. Those various issues which highlight the causes of intruder's, missing node and packet dropping all these issues are resolved from the existing methodology. So, this work gives new parameters for more precision in IDS. Fundamentally these works give more right and corrected measure by utilizing the effective use of information for node and improvement in PDR and Throughputs. By the above qualities the reliability in the system will be improved and effective system will be formed. By this packet, drops can be minimized and intruders can be recognized effectively and prove the high performance.
\end{abstract}

\section{Keywords}

Intrusion Detection System (IDS), Packet Delivery Ratio, Throughput, Routing Overhead

\section{INTRODUCTION}

Specially appointed Network is separated by its kind of correspondence bolstered qualities in two classifications wired or remote medium. Wired correspondence is area subordinate keeping in mind the remote correspondence is without area innovation. By considering gadget portability as a primary variable diverse system gives unmistakable utilities to clients. Any sort of system framework chips away at the premise of their transmission medium which can be any medium like radio waves which is a little range waves. Distinctive sort of transmission medium is GSM, MANET, WSN, Bluetooth, Zigbee et cetera. A remote versatile impromptu system imparts through radio transmission without the backing of altered directing foundation. Remote Ad hoc system is exceptionally straightforward and as a result of this, it has a parcel of requests in the military and other distinctive territories. This framework which utilized for transient correspondence to trade data goes under the class of Ad-hoc frameworks. With time the quantity of clients and their correspondence procedures are changing getting denser as per the necessities. It might be like mailing organization, vocabulary, ticket booking, military or business needs. In such districts radio transmission is possible and cell phones are similarly of compact sorts.

In remote transmission segment used for this are still subject to altered framework i.e. base station which can transmit the data up to a settled purpose of internment. Issues develop when such establishment is not open and the cost to do as such is also high for the supplier. By then the available organization could be passed on to the end customer without such base stations through uncommonly designated frameworks of impromptu systems. From the long stretch, the Ad hoc systems have been on the examination work area yet have as of late increased more consideration. For the last-mile issue the broadband remote access is depicted as the panacea in spite of the fact that the vision of flawless network and broadband remote web access is gorgeous to utilize, it is a long way from reality. For various managerial, specific and productive reasons, remote access frameworks general neglects to fulfill the insurance of steady, high-transmission limit, and direct organization. MANET is fundamentally called as a mix or gathering of various versatile hubs. When we discussing the any Ad hoc system for any versatile hub it is not particularly transfer on any brought together administrator. Any versatile or compact gadget depends on battery so that the principle point is that how to minimize the bundle drop. Along these lines, all the thought depends on expansion force of any gadget and utilize adequately with the goal that we can minimize the issue of bundle drop in the system. That is the reason any hub in the system moves out of its range then the connection between them is broken. This is required to minimize the issue of IDS.

\section{BACKGROUND}

MANET is alterably moving characteristic conditions which deal its security from various sorts of attacks. Interference is strikes which are deliberately planted as an undertaking which triggers after a specifically picked conditions and starts impacting the standard correspondence and makes the drops in the execution of the structure. The getting procedures are in advance said as frameworks and models. The qualification between both comes in execution and the kind of survey data associated as an information. Most of the IDS basically use aberrance based distinguishing proof as a piece of MANET. Interference activity in extraordinarily delegated framework is starts working after a specific strike delegated framework is starts working after a specific strike hit the working structure and in this way as showed by the outcomes attacks may be: Black hole, Routing circles, framework fragment, in tolerance, an absence of rest and foreswearing of an organization. As showed by their techniques they are accumulated as store hurting, produced a course, rushing, and wormhole, pack dropping, spoofing and threatening to flood. The convincing IDS is used to remember this initiating conditions advantageous by analyzing the framework data and recognize the source from which these attacks are propelled. 
Here the aggressor focuses towards imbuing, replaying or distorting the coordinating information for impacting the normal operation of framework and reasons superfluous weights and overhead. These center points should be recognized for further guaranteeing the centers and making both framework ambushes. Furthermore the data used to audit the MANET is not complete because of a sudden change in conditions. It is troublesome for a framework to isolate between the conventional and interference development in view of ceaseless flexibility of centers. Disregarding the way that there are a couple of essentials which should be considered for getting the intrusion from alterably changing conditions in MANET and given as:

1. An IDS must arrange the common and intruder movement with no modified infrastructural controlling core interests.

2. (ii)The Decision of sporadic action is group situated in nature and taken by the center points in a span especially.

3. Overheads associated with IDS should be minimized and should also keep up the separations whenever of time in the midst of the recognizable proof.

4. (iv)The transitional correspondence between IDS on particular center points must be secure to not allow strikes get the privilege to get access to such transmissions.

5. (v) An IDS essentially can't anticipate that any center point will be secure and in this way predictable appraisals of centers practices and data is performed.

6. (vi) An IDS can have the ability to take a decision by deficient data with a diminishment in high false alert rates.

7. (vii) It should also be prepared for finding the irregularities in practices and instantly lighting up interchange centers about this revelation.

Satisfying the above necessities needs a combined strategy of development displaying, framework and decision making. While taking building commitments in intrusion distinguishing proof it could be sorted in stand-alone, supportive and scattered and dynamic IDS. In stand-alone building plan, every one host has independent IDS with no cooperation from various center points in decision making. Second is scattered or supportive IDS in which each center point is having a pleasing IDS authorities which make the area and a short time later gives the acknowledgment to various centers exhaustively. The Third is different leveled IDS which is expected for multi-layer MANETS with a bundle head accountable for every one social occasion and imparts the information to the gathering head of other get-together and along these lines giving of information reasons authentic and fruitful decision. Shortly considering the decision making the order is of sort communitarian decision settling on and selfsufficient decision making. In people group decision making each center point joins in IDS decision making successfully and presents a strong protection against intrusions. While in decision making only a few of all center points are affirmed for finding the interferences.

\section{LITERATURE SURVEY}

In the midst of the most recent couple of years diverse examination articles had disseminated which surrenders the inconspicuous components to a specific level and in the wake of scrutinizing those some advanced strategies had been perceived.

In the paper [6], an intrusion revelation method provides portrayal system is shown for MANET. For doing this the procedure utilizes five key managed courses of action counts using assorted estimations. Their execution and evaluation are completed on the dataset which takes the data of movement conditions and convey ability case of the center points for strikes area. Routinely, after classifiers are tuned using crossendorsement, data from the same sorts of ambushes are open in all heading of work. The work had also broken down uniform and weighted estimations as classifiers using the tuning schedules for classifiers. Results finished up the execution based evaluation of classifier tuning.

In the paper [7], a layer joined structure of neural framework is given for intense intrusion ID and clearing. As a trial data set, the system uses KDD glass 1999 dataset. Close by some change with neural structures some connection is furthermore shown for checking the outcomes. The paper proposes two consolidated models named as Model A and Model B. Here the Model A considers all the ordinary tricks of planning datasets and the Model B considers only couple of characteristics which truly makes the convincing responsibility to request process for computation time diminishment. The delayed consequences of proposed modelbased procedure of proposed work will show its capability.

In the paper [8], another model of enhanced flexible affirmation (EAACK) based IDS is proposed for MANET. As a matter of first importance, the work had examined distinctive existing arrangements for intrusion revelation and clearing which later on changed by one means or another for better results. As differentiated and diverse approaches the EAACK is demonstrating higher revelation rates. The approach is especially planned to handle a couple issues of standard systems. These issues are false unfortunate behavior, compelled transmission power effect et cetera. It is having three essential parts named as ACK, secure ACK and rambunctiousness report confirmation. At the key level of evaluation, the system is showing its profitability over the present gatekeeper pooch part.

The paper [9] proposed a novel RAODV based improved intrusion recognizable proof framework for higher security. The system is engaged around trust-based structures used for securing the senders IP and having stamped verification as an affirmation for further occupations. The illustrated coordinating tradition will be considered few of the directing components for separating the center points as gate crasher. For the most part, it took estimations of stopping up, imperfect center points and capably developing topologies. The work had made an IDS checking center which is having some additional information for taking care of with adjusted headers. The RAODV included two sorts of control groups: Reliable Route Discovery Unit (RRDU) and RRDU Reply in existing traditions. Continuing with the above examination and outlines the paper [12] puts a strong study on the present IDS with their ideal circumstances and burdens. Its examinations every one of the segments for and DS used n remote frameworks and measures their execution in different working conditions. The paper finally settles on a portrayal by 
which decision of IDS is made straightforward for various circumstances.

The paper [10] focuses on interference foresight security procedures like encryptions and approvals which reduce the risk up to a specific purpose of repression, however, can't have the ability to thoroughly overcome this. The paper moreover proposes a novel quantitative intrusion recognizable proof using behavioral idiosyncrasy based revelation. The proposed system is versatile, component, and overwhelming to support constantly convey ability based environment. Entertainment of proposed work with AODV tradition is also shown in the paper which makes the recognizing verification of results straightforward an intense. The procedure uses an IDs administrator to make the records of assorted transmission and exchanging with complete versatility scope. These authorities run freely and watch activities of the customer and structure and correspondence practices inside their radio degree for perceiving the malevolent activities. Unmistakable makers had focused on different response for improving the acknowledgment rates and decreasing the false irritating. In a way to do as such the paper displays novel IDS centered around genetic computations. In the proposed work the inherited counts is used for gathering the portrayal realizes close perfect time which makes the early disclosure and clearing. The work had in like manner uses relationship framework to perceive the most basic characteristics of framework relationship in flexible off the cuff frameworks. With innate estimations, the feasible IDS will depend on upon its adaptable representation of the standards and effective health works that can be associated. At the appraisal viewpoint, the procedure is exhibiting its best similarly as various execution component changes and higher revelation rates.

The paper [11] presents an IDS centered around data mining based techniques, for instance, Id3 by which decision tree can be melded and recognizable proof rates can be made progress. The work had similarly proposed a particular Id 3 computation in which TTL qualities are tuned to work with the classifiers. Here the TTL worth is utilized to get a joined response for use Id3 estimation of decision trees and ID trees in the classifier of IDS. At the use level, the work had used a surely understood mining gadget Weka and KDD compartment 1999 datasets. As needs be, in the wake of looking at the changed part of a convincing intrusion revelation structure, this work recognized few issues which stay unsolved. These issues will accept a vital part in making the nearby perfect system for exhibiting the best results. Also, the work had needed to effectively inspect the impacts of changed thoughts on ordinary IDS and measured.

\section{PROBLEM STATEMENT \& PROPOSED SOLUTION}

The Intrusion technique is a kind of undesirable activity that causes the framework execution corruption and in this way should be distinguished in right on time periods of transmissions. As of the earth of extraordinarily named framework is remote and the tradition used to help movability is in like manner lightweight, the security part proposed to stay away from such strikes is not adequate. They are complicated and not adequately exact to keep those strikes like Dos, analyzing, drops et cetera. In the wake of looking at the changed examination articles, it creates the impression that copying issue stays unaddressed if there ought to be an event of IDS and is having a wide zone of work here.
Transmission Overhearing: For dismembering the neighbor interloper's direct some checking or central force center points or every one neighbor center can here the transmission of its neighbor center. False Misbehavior Report and Cooperative Nature Detection: Some of the centers can grab that their working and transmission arrangements are secure anyway they are dropping the groups or decreasing the framework execution. So it should be recognized in right on time periods of IDS.

Affirmation Count: Total Acknowledgment number can be taken for Nodes Trust Index by which on the reason of affirmation sent and get a trust record of centers is structure whose higher qualities exhibits the right carrying on centers.

Unwavering quality of Route: For each productive transmission or package sent and get extent the reliability obviously can be extended which exhibits that the course exists and is not serving as data drops or gate crashers helpfulness. Its examinations every one of the segments for and DS used in remote frameworks and measures their execution in differing working conditions.

Jump Counts Filter: Number of ricochet checks can in like manner be taken as a condition for interference in light of the way that for an immense framework in which convenience is high it is acknowledged that the greatest hop implies a package should not more than 30 . In any case, here and there it should be more so for such bundles the tradition requires intruder's behavior however in bona fide it is a true blue one.

\section{PROPOSED ALGORITHM}

Algorithm suggested

$(\mathrm{N}, \mathrm{i})$

\{

DECLARE ACK3, ACK ,Data Packet

DECLARE Node, Src, Time Out, T

Destination $=$ SRC $-\&$ get Data Packet

//Source expect ACK from destination

If (ACK! $=$ NULL)

Else if (ACK3!=NULL)

Else

\{

Print "Source received ACK from destination successfully"

\}

1

Print "Source received ACK from neighbour node 3"

\}

\{

Print "Malicious Node" 


\section{GENERATED OUTCOMES}

In this work, it demonstrates the value of result with the assistance of proposed technique. By utilizing NS2 to judge the execution of a few metric are there to assess. By utilizing this re-enactment it proposed this work will utilize uncommon presentation measurements for demonstrating the foreseen results. In this work utilizing X diagram utility of NS2 results are plotted.

\subsection{PDR (Packet Delivery Ratio) Graph}

PDR is the part of the measure of bundles recognized at the objective hub from the quantity of parcels sent from the proposed hub. In this way, by these outcomes when PDR is uniform and high the execution is best for any hub.

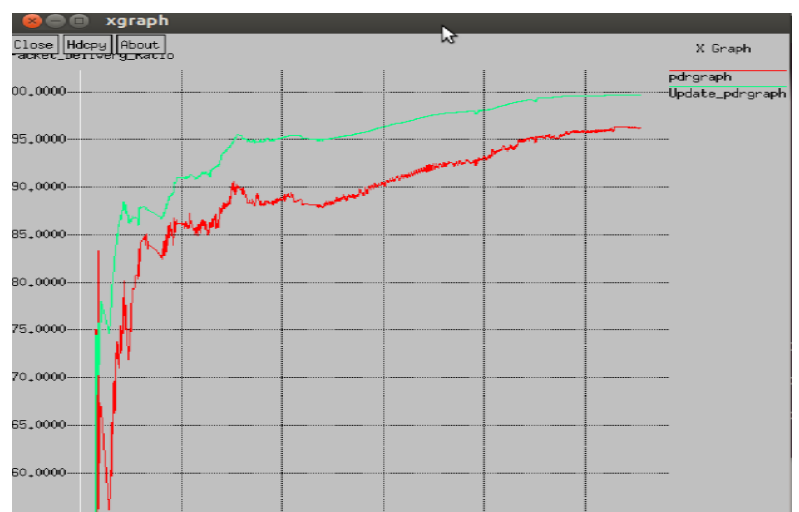

Graph 6.1 :Comparison of PDR Ratio of Proposed and Existing

Graph review : In proportion is required as a part of PDR. It depends on the measure of parcel send is gotten in the equivalent sum. In this way, in the best condition, it ought to be high and uniform as could be expected under the circumstances. When we judging the present work of the above chart it recommends the outcome as a proposed technique produce the preferred PDR proportion over the more established methodologies.

\subsection{Throughput}

In throughput ii is a part of the medium limit which is utilized for transmission, for that consider an objective at the underlying phase of the recreation. For instance the information at whatever point needs to exchange however it is required that the information bundles conveyed accurately to the objective.

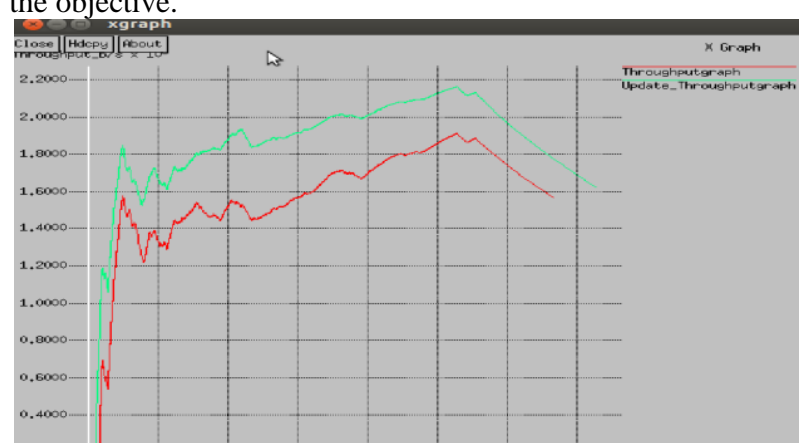

Graph 6.2 : Evaluation of Throughput of Proposed and Existing Throughput
Graph review: In parcel, conveyance proportion is required to discover the execution of the techniques utilized as a part of throughput. It depends on the measure of parcel send is gotten in the equivalent sum. In this way, in the best condition, it ought to be high and uniform as could be expected under the circumstances. When we judging the present work of the above chart it recommends the outcome as a proposed technique produce the preferred Throughput proportion over the more established methodologies.

\subsection{Routing Overhead Load (ROL)}

ROL is the part of an entire number of the steering hub which has the parcel which is equivalent to the aggregate of got information bundles at the target end. Along these lines, the aggregate sum of element parcel produced for each information movement transmission (in bits). Presently, when we consider as far as the additional heap incident while executing the prescribed method when contrasted with the standard convention stack for the framework.Mulling over the accompanying components

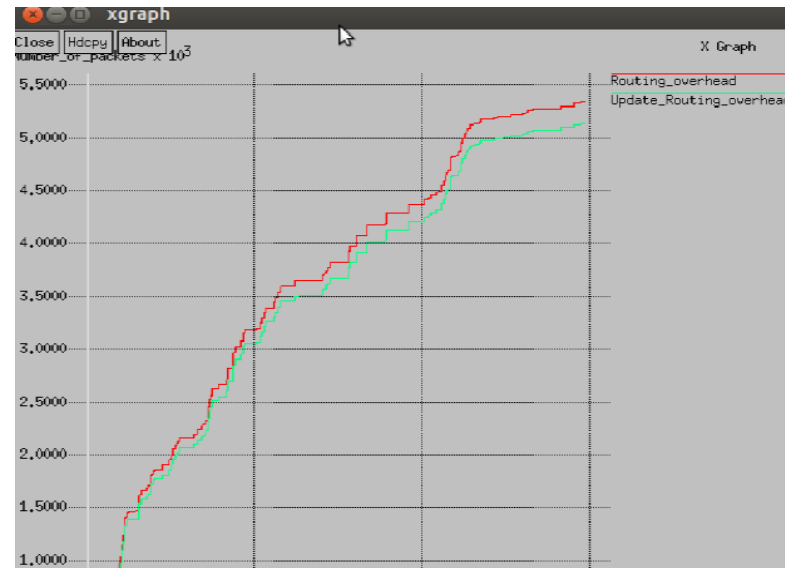

Graph 6.3: Comparison of Routing Overhead of Proposed and Existing Overhead

Graph review: The above result confirms its outcomes by minimizing the directing overhead which is associated with the prescribed technique. This chart additionally demonstrates that the entanglement in the current strategy is less when contrasted with the proposed technique. 


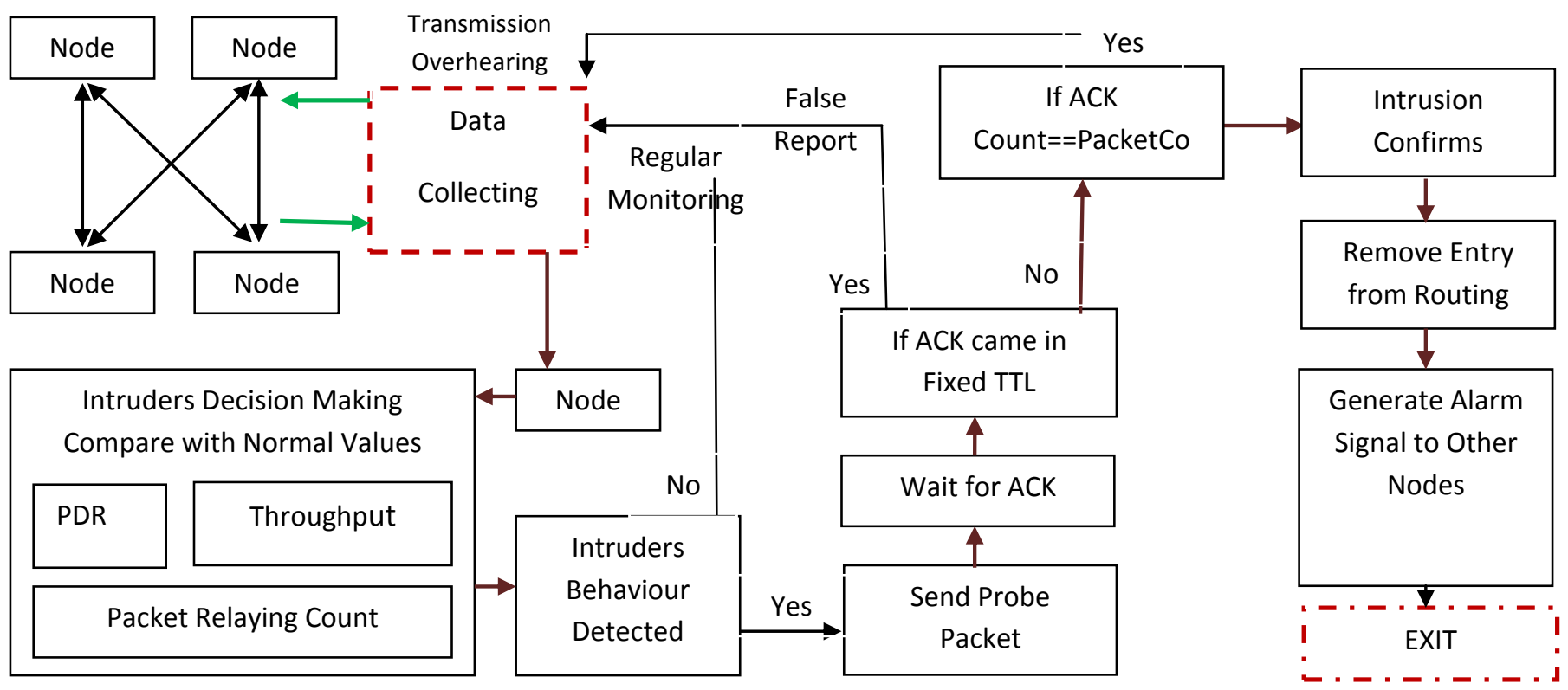

Figure 1: anticipated Intrusion recognition System With usual Monitoring of Nodes Behaviour

\section{CONCLUSION}

MANET is the short range radio framework used for passing on with no infrastructural parts. Here the center points will serve the complete value of switches. For transmitting the packs to expelled zone the midway neighbor's center points are used for transmitting the bundles. For this, an effective coordinating tradition is required for giving the perfect guiding. Security issues are of prime worry in the remote framework and in this manner the encryption count is used. In the meantime, in the case of intruders this security estimation fails to get those gate crashers attack which is organized or sudden shows unusual action works out. For the most part, the intrusion disclosure systems are engaged around data examination and in this way most of the times their time report are false and not correct. Along these lines, this work proposes a novel IDS cantered around a couple of variables which interminably impacts the behavior of the center. If these segments are seen over a period of time than interlopers behavior is taken after out accurately. At the interpretive level of result examination of proposed philosophy with existing instrument, the prescribed work exhibits its adequacy and accuracy. Along these lines, this work proposes an upgraded IDS answer for crushing these issues using. The work uses a standard, which hears the transmission of various center points as well. These transmissions had a quality diverged from the standard edge regard with request veritable and getting into devilishness center points. At the appraisal point of view, the paper in like manner gives a couple results execution parameters examination and connection with existing structures. This work showed coherently that the proposed strategy is enough upgrading the framework

execution and is better than any of the standard interference acknowledgment approach. In like manner the procedure makes the framework lives for more term in light of its less imperativeness usage and low overheads.

\section{ACKNOWLEDGMENT}

After the completion of this project, work is not enough to express my feeling about all those who helped me to reach my goal; feeling above this my indebtedness to The Almighty for providing me this moment in life.

It's a great pleasure and moment of immense satisfaction for me to express my profound gratitude to Mrs. Rupali Bhartiya, Reader, Computer Science and Engineering Department SVITS-Indore, whose constant encouragement enabled me to work enthusiastically. Their perpetual motivation, patience and excellent expertise in discussion during the progress of the dissertation work have benefited me to an extent, which is beyond expression.

I express my sincere thanks and gratitude to Dr. Anand Rajavat, HOD, Computer Science and Engineering Department, SVITS-Indore, who took care about imparting expert knowledge for design and development of the project.

\section{REFERENCES}

[1] Anant R. More, Vikas N. Nandgaonkar, Dr.ManojNagmode, Pramod P. Patil "ID3 Algorithm for Intrusion Detection" International Conference on Recent Trends in engineering \& Technology 2013(ICRTET'2013).

[2] Ahmed Youssef and Ahmed Emam "Network Intrusion Detection using Data Mining and Network.Behavior Analysis" International Journal of Computer Science \& Information Technology (IJCSIT) Vol 3, No 6, Dec 2011.

[3] AnazidaZainal, MohdAizainiMaarof and SitiMariyamShamsuddin "Data Reduction and Ensemble Classifiers in Intrusion Detection" in 2008 IEEE.

[4] Chau M., Xu J.J. and Chen H. (2002) National Conference on Digital Government Research, 271-275.

[5] Devaraju .S, Ramakrishnan .S "Detection of Accuracy for Intrusion Detection System using Neural Network International Journal of Computer Applications (0975 8887) Classifier" International Journal of Emerging 
Technology and Advanced Engineering( ISSN 22502459 (Online), An ISO 9001:2008 Certified Journal, Volume 3, Special Issue 1, January 2013).

[6] Devendrakailashiya, Dr. R.C. Jain "Improve Intrusion Detection Using Decision Tree with Sampling" in IJCTA | MAY-JUNE 2012.

[7] GuangqunZhai, Chunyan Liu "Research and Improvement on ID3 Algorithm in Intrusion Detection System" in 2010 IEEE.

[8] Jorge Blasco, Agustin Orfila, Arturo Ribagorda "Improving Network Intrusion Detection by Means of Domain-Aware Genetic Programming" DOI 10.1109/ARES.2010.53 in IEEE 2010.

[9] Joshi .S.A, VarshaS.Pimprale "Network Intrusion Detection System (NIDS) based on Data
Mining"International Journal of Engineering Science and Innovative Technology (IJESIT) Volume 2, Issue 1, January 2013.

[10] Mohd. JunedulHaque, Khalid.W. Magid, Nisar Hundewale "An Intelligent Approach for Intrusion Detection Based on Data Mining Techniques" in 2012 IEEE.

[11] YacineBouzida, Frederic Cuppens "Neural networks vs. decision trees for intrusion detection" in 2011.SIGMOD Rec-ord, 30 (4), 25-34.

[12] YacineBouzida, Frederic Cuppens "Neural networks vs. decision trees for intrusion detection" in 2011. SIGMOD Rec-ord, 30 (4), 25-34. Volume 90 - No 12, March 2014 